\title{
Finite-time synchronization for a class of dynamical complex networks with nonidentical nodes and uncertain disturbance
}

DOI:

10.1007/s11424-018-8141-5

\section{Document Version}

Accepted author manuscript

Link to publication record in Manchester Research Explorer

Citation for published version (APA):

Li, Q., Guo, J., Sun, C., Wu, Y., \& Ding, Z. (2019). Finite-time synchronization for a class of dynamical complex networks with nonidentical nodes and uncertain disturbance. Journal of Systems Science and Complexity, 32(3), 818-834. https://doi.org/10.1007/s11424-018-8141-5

Published in:

Journal of Systems Science and Complexity

\section{Citing this paper}

Please note that where the full-text provided on Manchester Research Explorer is the Author Accepted Manuscript or Proof version this may differ from the final Published version. If citing, it is advised that you check and use the publisher's definitive version.

\section{General rights}

Copyright and moral rights for the publications made accessible in the Research Explorer are retained by the authors and/or other copyright owners and it is a condition of accessing publications that users recognise and abide by the legal requirements associated with these rights.

\section{Takedown policy}

If you believe that this document breaches copyright please refer to the University of Manchester's Takedown Procedures [http://man.ac.uk/04Y6Bo] or contact uml.scholarlycommunications@manchester.ac.uk providing relevant details, so we can investigate your claim.

\section{OPEN ACCESS}




\title{
Finite-Time Synchronization for a Class of Dynamical Complex Networks with Nonidentical Nodes and Uncertain Disturbance*
}

\author{
LI Qingbo · GUO Jin · SUN Changyin · WU Yuanyuan · DING Zhengtao
}

DOI: $10.1007 / \mathrm{s} 11424-018-8141-5$

Received: 3 May 2018 / Revised: 31 May 2018

(c) The Editorial Office of JSSC \& Springer-Verlag GmbH Germany 2019

\begin{abstract}
This paper investigates the finite-time synchronization for a class of linearly coupled dynamical complex networks with both nonidentical nodes and uncertain disturbance. A set of controllers are designed such that the considered system can be finite-timely synchronized onto the target node. Based on the stability of the error equation, the Lyapunov function method and the linear matrix inequality technique, several sufficient conditions are derived to ensure the finite-time synchronization, and applied to the case of identical nodes and the one without uncertain disturbance. Also the adaptive finite-time synchronization is discussed. A numerical example is given to show the effectiveness of the main results obtained.
\end{abstract}

Keywords Disturbance, dynamical complex networks, finite-time synchronization, nonidentical nodes.

\footnotetext{
LI Qingbo

School of Automation and Electrical Engineering, University of Science and Technology Beijing, Beijing 100083,

China; College of Mathematics and Information Science, Zhengzhou University of Light Industry, Zhengzhou 450002, China. Email: qbliyy@163.com.

GUO Jin (Corresponding author)

School of Automation and Electrical Engineering, University of Science and Technology Beijing, Beijing 100083,

China; Key Laboratory of Knowledge Automation for Industrial Processes, Ministry of Education, Beijing 100083, China. Email: guojin@ustb.edu.cn.

SUN Changyin

School of Automation, Southeast University, Nanjing 210096, China. Email: cysun@seu.edu.cn.

WU Yuanyuan

College of Electric and Information Engineering, Zhengzhou University of Light Industry, Zhengzhou 450002, China. Email: wyuanyuan82@163.com.

DING Zhengtao

School of Electrical and Electronic Engineering, University of Manchester, Manchester M13 9PL, UK.

Email: zhengtao.ding@manchester.ac.uk.

* This research was supported in part by the National Natural Science Foundation of China under Grant Nos. 61773054, 61603350 and 61501407, and in part by the Fundamental Research Funds for the Central Universities of USTB (FRF-TP-17-039A2).

$\diamond$ This paper was recommended for publication by Editor ZHAO Yanlong.
} 


\section{Introduction}

In the past few decades, complex networks have gained a lot of attention in various fields of science and humanity worldwide, such as food-webs, ecosystems, metabolic pathways, the World Wide Webs and so on. Their control and synchronization have been deeply investigated, and various relevant theoretical results have been established ${ }^{[1-7]}$. Also many effective control approaches have been proposed, such as adaptive control ${ }^{[8,9]}$, intermittent control ${ }^{[10-13]}$, impulsive control ${ }^{[14-17]}$, slide mode control ${ }^{[18-20]}$ and sampled-data control ${ }^{[21,22]}$, et al.

It is frequently encountered that many significant differences exist in the relevant individual nodes. For example, in power systems, because the generators and loads are connected to buses that are interconnected via transmission lines in a network structure, the power systems can be considered as a dynamical network with nonidentical nodes ${ }^{[23]}$. When the dynamics of nodes in a complex network are nonidentical, the synchronization problems become complicated and more challenging than the case of identical nodes. By use of free matrices, [23] considered the case of synchronizing to both a common equilibrium solution of all isolated nodes and the average state trajectory with nonidentical nodes. Combining the local intermittent controller with the open-loop controller, [24] established several exponential synchronization criteria for a class of complex networks with nonidentical nodes. In [25], the pinning cluster synchronization of complex dynamical networks with time-delayed coupling and dynamic nonidentical nodes was obtained. [26] studied the finite-time synchronization problem for linearly coupled complex networks with discontinuous nonidentical nodes.

On the other hand, more and more attention has been paid to the study of complex networks with perturbations because of the wide application ${ }^{[27-30]}$. [27] investigated globally exponential synchronization for linearly coupled neural networks with time-varying delay and impulsive disturbances. The derived sufficient condition was closely related with the time delay, impulse strengths, average impulsive interval, and coupling structure of the systems. [28] addressed the scheme of cluster synchronization of overlapping uncertain complex networks with time-varying impulse disturbances. In [29], the cluster synchronization problem of coupled complex networks with uncertain disturbance was considered under an adaptive fixed-time control strategy.

Most of the related research focuses on either nonidentical nodes or uncertain disturbance. Hence it is very necessary and important, with profound theoretical and practical significance, to investigate the finite-time synchronization of complex networks subject to both nonidentical nodes and uncertain disturbance. However, two difficulties have to be faced: (i) What conditions should be proposed which are applicable to general complex networks with both nonidentical nodes and uncertain disturbance and are easy to be verified? (ii) Which kind of controller should be designed such that the nonidentical nodes and the uncertain disturbance can be well dealt with? This paper aims to overcome these two difficulties and achieve finite-time synchronization for a class of linearly coupled complex networks with both nonidentical nodes and uncertain disturbance, and further enrich the theoretical results of finite-time synchronization. The main contributions in this paper can be summarized as follows: 1) A novel discontinuous controller is designed for a class of heterogeneous networks with uncertain disturbance and the controller 
can overcome the influence of heterogeneous and uncertain disturbance simultaneously on the finite-time synchronization of the network; 2) Several criteria have been derived to check the finite-time synchronization of the considered networks. Different from most of the existing results, the obtained finite-time synchronization conditions are represented in the form of linear matrix inequalities, and easy to be verified; 3) The adaptive finite-time synchronization of the heterogeneous networks are addressed.

Firstly, in order to make the results obtained more easily verified and applied in practice, a model of the complex network with both nonidentical nodes and uncertain disturbance is established. After that, a set of controllers are designed. Based on the finite-time stability for the error equation, the Lyapunov function method and the linear matrix inequality technique, it is shown that the complex network considered can be finite-timely synchronized onto any isolated driving node. Secondly, several sufficient criteria are obtained to guarantee the synchronization goal, and applied to the case of identical nodes and the one without uncertain disturbance. Meanwhile, the adaptive finite-time synchronization is also discussed.

The remainder of this paper is organized as follows. In Section 2, the complex network model with both nonidentical nodes and uncertain disturbance is formulated and the finitetime synchronization problem is introduced. The finite-time synchronization conditions are obtained in Section 3. Section 4 considers the finite-time synchronization by the adaptive control method. In Section 5, a numerical example is provided to illustrate the validity of the method proposed. Some conclusions are made in Section 6, together with some potential future study.

\section{Problem Formulation}

Consider an array of nonlinear systems with the linear and diffusive coupling consisting of $N$ nonidentical nodes in which each node is an $n$-dimensional dynamical system with uncertain disturbance as follows

$$
\dot{x}_{i}(t)=f_{i}\left(t, x_{i}(t)\right)+h_{i}\left(t, x_{i}(t)\right)+c \sum_{j=1}^{N} G_{i j} \Gamma x_{j}(t),
$$

where $x_{i}(t)=\left[x_{i 1}(t), x_{21}(t), \cdots, x_{i n}(t)\right]^{\mathrm{T}} \in \mathbb{R}^{n}$ is the state vector of the $i$ th dynamical node; $f_{i}\left(t, x_{i}(t)\right): \mathbb{R}^{+} \times \mathbb{R}^{n} \rightarrow \mathbb{R}^{n}$ is a smooth nonlinear vector fields describing the modal selfdynamics, and $h_{i}\left(t, x_{i}(t)\right): \mathbb{R}^{+} \times \mathbb{R} \rightarrow R$ is the uncertain disturbance, $i=1,2, \cdots, N ; c>$ 0 is a constant and denotes the coupling strength of the whole complex network, and $\Gamma=$ $\operatorname{diag}\left(\gamma_{1}, \gamma_{2}, \cdots, \gamma_{n}\right) \in \mathbb{R}^{n \times n}$ is the inner-coupling matrix, which is used to illustrate the way of linking the components in every pair vector of nodes with $\gamma_{i} \geq 0 ; G=\left(G_{i j}\right)_{N \times N}$ is the constant coupling configuration matrix which represents the topological structure and may be defined to be diffusive, i.e., $G_{i j} \geqslant 0(i \neq j)$ if there exists a directed connection from node $j$ to node $i$; otherwise, $G_{i j}=0$ and $G_{i i}=-\sum_{j=1, j \neq i}^{N} G_{i j}$. Here, the coupling matrices $G$ is nor required to be symmetric or irreducible. 
We refer to the system (2) with $\left.x_{0}\right|_{t=0}=x_{0}(0)$ as the driven dynamical node of (1)

$$
\dot{x}_{0}(t)=f_{0}\left(t, x_{0}(t)\right)+h_{0}\left(t, x_{0}(t)\right) .
$$

Definition 2.1 (see [31]) The complex network (1) is said to be synchronized in finite time if there exist a designed feedback controller and a constant $t^{*}>0$, which often depends on the initial state vector value $X(0)=\left(x_{1}^{\mathrm{T}}(0), x_{2}^{\mathrm{T}}(0), \cdots, x_{N}^{\mathrm{T}}(0)\right)^{\mathrm{T}}$, such that

$$
\lim _{t \rightarrow t^{*}}\left\|x_{i}(t)-x_{0}(t)\right\|=0 \text {. }
$$

Then the synchronization performance of drive-response network are achieved in a finite-time, i.e.,

$$
\left\|x_{i}(t)-x_{0}(t)\right\|=0, \quad t>t^{*}, \quad i=1,2, \cdots, N .
$$

This paper aims to design feedback controllers for the complex network (1) to achieve the finite-time synchronization.

Assumption 2.1 (see [32]) There exists a uniformly symmetric positive definite matrix $L=\operatorname{diag}\left(l_{1}, l_{2}, \cdots, l_{n}\right)$ such that $f_{i}(t, x)$ satisfies

$$
(y-x)^{\mathrm{T}}\left(f_{i}(t, y)-f_{i}(t, x)\right) \leq(y-x)^{\mathrm{T}} L(y-x), \quad i=1,2, \cdots, N
$$

for all $x, y \in \mathbb{R}^{n}$ and $t \geq 0$.

Assumption 2.2 (see [30]) There exists a time-varying function $\mu(t) \geq 0$ such that

$$
\|\left(f_{i}(t, x)-f_{0}(t, x) \| \leq \mu(t), \quad i, j=1,2, \cdots, N .\right.
$$

Assumption 2.3 The uncertain disturbances $h_{i}\left(t, x_{i}(t)\right)$ are continuous at $t, x_{i}(t) \geq 0$, and bounded by a given non-negative number $h_{\max }$, that is,

$$
\left|h_{i}\left(t, x_{i}(t)\right)\right| \leq h_{\max }, \quad i=0,1, \cdots, N
$$

Remark 2.2 Assumption 2.1 is satisfied with chaotic oscillators and Rössler's systems and so on. Assumption 2.2 and Assumption 2.3 impose restrictions on the activation function, and they are widely used in literatures ${ }^{[23-26]}$.

\section{Finite-Time Synchronization}

In this section, we design controllers for the finite-time synchronization of the complex network (1). For this, the controllers $u_{i}(t) \in R^{n}$ are constructed as

$$
u_{i}(t)=-d_{i} e_{i}(t)-\eta(t) \overline{\operatorname{sign}}\left(e_{i}(t)\right)-k \operatorname{sign}\left(e_{i}(t)\right)\left|e_{i}(t)\right|^{\beta}, \quad i=1,2, \cdots, N,
$$

where $d_{1}, d_{2}, \cdots, d_{N}$ are positive constants to be determined, $k>0$ is a constant, $\overline{\operatorname{sign}}\left(e_{i}(t)\right)=$ $\left(\operatorname{sign}\left(e_{i 1}(t)\right), \operatorname{sign}\left(e_{i 2}(t)\right), \cdots, \operatorname{sign}\left(e_{i n}(t)\right)\right)^{\mathrm{T}}, \operatorname{sign}\left(e_{i}(t)\right)=\operatorname{diag}\left(\operatorname{sign}\left(e_{i 1}(t)\right), \operatorname{sign}\left(e_{i 2}(t)\right), \cdots\right.$, $\left.\operatorname{sign}\left(e_{i n}(t)\right)\right),\left|e_{i}(t)\right|^{\beta}=\left(\left|e_{i 1}(t)\right|^{\beta},\left|e_{21}(t)\right|^{\beta}, \cdots,\left|e_{i n}(t)\right|^{\beta}\right)^{\mathrm{T}}$, and the real number $\beta$ follows $0 \leq \beta<1$. 
Then the controlled complex dynamical network is given by

$$
\dot{x}_{i}(t)=f_{i}\left(t, x_{i}\right)+h_{i}\left(t, x_{i}(t)\right)+c \sum_{j=1}^{N} G_{i j} \Gamma x_{j}(t)+u_{i}(t), \quad i=1,2, \cdots, N .
$$

By defining the synchronization errors $e_{i}(t)=x_{i}-x_{0}, F_{i}(t)=f_{i}\left(t, x_{i}\right)-f_{0}\left(t, x_{0}\right)$ and $H_{i}\left(t, e_{i}(t)\right)=$ $h_{i}\left(t, x_{i}(t)\right)-h_{0}\left(t, x_{0}(t)\right)$, the error dynamical system can be represented by

$$
\dot{e}_{i}(t)=F_{i}\left(t, e_{i}\right)+H_{i}\left(t, e_{i}(t)+c \sum_{j=1}^{N} G_{i j} \Gamma e_{j}+u_{i}(t), \quad i=1,2, \cdots, N .\right.
$$

Lemma 3.1 (see [33, 34]) Assume that a continuous, differentiable, positive-definite function $V(t):[0,+\infty) \rightarrow[0,+\infty)$ satisfies

$$
\frac{d V(t)}{d t} \leq-\eta V^{\alpha}(t), \quad \forall t \geq t_{0}, \quad V\left(t_{0}\right) \geq 0
$$

where $\eta>0$ and $0<\alpha<1$ are two constants. For any given $t_{0}$, one can have

$$
V^{1-\alpha}(t) \leq V^{1-\alpha}\left(t_{0}\right)-\eta(1-\alpha)\left(t-t_{0}\right), \quad t_{0} \leq t \leq t_{1}
$$

and $V(t) \equiv 0, t>t_{1}=t_{0}+\frac{V^{1-\alpha}\left(t_{0}\right)}{\eta(1-\alpha)}$.

Lemma 3.2 (see [35]) For matrices A, B, C,D with appropriate dimensions and a scalar $\alpha$, the following assertions hold.

1) $(\alpha A) \otimes B=A \otimes(\alpha B)$;

2) $(A+B) \otimes C=A \otimes C+B \otimes C$;

3) $(A \otimes B)(C \otimes D)=(A C) \otimes(B D)$;

4) $(A \otimes B)^{\mathrm{T}}=A^{\mathrm{T}} \otimes B^{\mathrm{T}}$, where $\otimes$ is the Kronecker product.

Lemma 3.3 (see [36]) Suppose that $a_{i} \geq 0$ for $i=1,2, \cdots, n, 0<p \leq 1$ and $0<q<2$, it follows that $\left(\sum_{i=1}^{n} a_{i}\right)^{p} \leq \sum_{i=1}^{n}\left(a_{i}\right)^{p}$ and $\sum_{i=1}^{n}\left(a_{i}\right)^{q} \geq\left(\sum_{i=1}^{n} a_{i}^{2}\right)^{q / 2}$.

Theorem 3.4 Consider the complex network (1) under the set of controllers (6). If Assumption 2.1, Assumption 2.2 and Assumption 2.3 hold, and

$$
\eta(t) \geq \mu(t)+2 h_{\max }, \quad I_{N} \otimes L+c\left(G^{s} \otimes \Gamma\right)-D \otimes I_{n}<0
$$

with $D=\operatorname{diag}\left\{d_{1}, d_{2}, \cdots, d_{N}\right\}>0, G^{s}=\left(G+G^{\mathrm{T}}\right) / 2, L$ being given by Assumption 2.1, then (7) can be synchronized in a finite time with

$$
t^{*}=\frac{V(0)^{\frac{1-\beta}{2}}}{\left.2^{\frac{\beta-1}{2}} k(1-\beta)\right)}
$$

where $V(0)=\frac{1}{2} \sum_{i=1}^{N} e_{i}^{\mathrm{T}}(0) e_{i}(0)$ and $e_{i}(0)$ is the initial condition of $e_{i}(t)=x_{i}(t)-x_{0}(t)$ for $i=1,2, \cdots, N$.

Springer 
Proof For (8), a Lyapunov function is constructed by

$$
V(t)=\frac{1}{2} \sum_{i=1}^{N} e_{i}^{\mathrm{T}}(t) e_{i}(t)
$$

whose derivative along the trajectory of System (8) can be calculated as

$$
\begin{aligned}
\dot{V}(t)= & \sum_{i=1}^{N} e_{i}^{\mathrm{T}}(t) \dot{e}_{i}(t) \\
= & \sum_{i=1}^{N} e_{i}^{\mathrm{T}}(t)\left\{F_{i}\left(t, e_{i}(t)\right)+H_{i}\left(t, e_{i}(t)\right)+c \sum_{j=1}^{N} G_{i j} \Gamma e_{j}(t)+u_{i}(t)\right\} \\
= & \sum_{i=1}^{N} e_{i}^{\mathrm{T}}(t)\left\{-d_{i} e_{i}(t)-\eta(t) \overline{\operatorname{sign}}\left(e_{i}(t)\right)-k \operatorname{sign}\left(e_{i}(t)\right)\left|e_{i}(t)\right|^{\beta}\right\} \\
& +I_{1}(t)+I_{2}(t)+I_{3}(t),
\end{aligned}
$$

with $I_{1}(t)=c \sum_{i=1}^{N} e_{i}^{\mathrm{T}}(t) \sum_{j=1}^{N} G_{i j} \Gamma e_{j}(t), I_{2}(t)=\sum_{i=1}^{N} e_{i}^{\mathrm{T}}(t) H_{i}\left(t, e_{i}(t)\right)$, and $I_{3}(t)=\sum_{i=1}^{N}$ $e_{i}^{\mathrm{T}}(t) F_{i}\left(t, e_{i}(t)\right)$.

From Lemma 3.2 and noticing that $\Gamma$ is a diagonal matrix, we have

$$
\begin{aligned}
I_{1}(t) & =c e^{\mathrm{T}}(t)(G \otimes \Gamma) e(t) \\
& =c e^{\mathrm{T}}(t) \frac{G \otimes \Gamma+(G \otimes \Gamma)^{\mathrm{T}}}{2} e(t) \\
& =c e^{\mathrm{T}}(t)\left(G^{s} \otimes \Gamma\right) e(t) .
\end{aligned}
$$

By use of (5) in Assumption 2.3, one can get

$$
\begin{aligned}
I_{2}(t) & =\sum_{i=1}^{N} e_{i}^{\mathrm{T}}(t)\left(H_{i}\left(t, x_{1}(t)\right)-H_{0}\left(t, x_{0}(t)\right)\right) \\
& \leq \sum_{i=1}^{N}\left\|e_{i}(t)\right\|_{2}\left\|H_{i}\left(t, x_{1}(t)\right)-H_{0}\left(t, x_{0}(t)\right)\right\|_{2} \\
& \left.\left.\leq \sum_{i=1}^{N}\left\|e_{i}(t)\right\|_{2}\left(\| H_{i}\left(t, x_{1}(t)\right)\right)\right)\left\|_{2}+\right\| H_{0}\left(t, x_{0}(t)\right) \|_{2}\right) \\
& \leq \sum_{i=1}^{N}\left\|e_{i}(t)\right\|_{2}\left(2 h_{\max }\right) .
\end{aligned}
$$

Also it can be seen that

$$
\begin{aligned}
I_{3}(t) & =\sum_{i=1}^{N} e_{i}^{\mathrm{T}}(t)\left(f_{i}\left(t, x_{i}(t)\right)-f_{0}\left(t, x_{0}(t)\right)\right) \\
& =\sum_{i=1}^{N} e_{i}^{\mathrm{T}}(t)\left(\left(f_{i}\left(t, x_{i}(t)\right)-f_{i}\left(t, x_{0}(t)\right)\right)+\left(f_{i}\left(t, x_{0}(t)\right)-f_{0}\left(t, x_{0}(t)\right)\right)\right)
\end{aligned}
$$




$$
=I_{31}(t)+I_{32}(t)
$$

with $I_{31}(t)=\sum_{i=1}^{N} e_{i}^{\mathrm{T}}(t)\left(f_{i}\left(t, x_{i}(t)\right)-f_{i}\left(t, x_{0}(t)\right)\right)$ and $I_{32}(t)=\sum_{i=1}^{N} e_{i}^{\mathrm{T}}(t)\left(f_{i}\left(t, x_{0}(t)\right)-\right.$ $\left.f_{0}\left(t, x_{0}(t)\right)\right)$. From (3) in Assumption 2.1, it is known that

$$
I_{31}(t) \leq \sum_{i=1}^{N} e_{i}^{\mathrm{T}}(t) L e_{i}(t)=e^{\mathrm{T}}(t) I_{N} \otimes L e(t) .
$$

By (4) in Assumption 2.2, one can get

$$
\begin{aligned}
I_{32}(t) & \leq \sum_{i=1}^{N}\left\|e_{i}(t)\right\|_{2}\left\|f_{i}\left(t, x_{0}(t)\right)-f_{0}\left(t, x_{0}(t)\right)\right\|_{2} \\
& =\sum_{i=1}^{N}\left\|e_{i}(t)\right\|_{2} \mu(t) .
\end{aligned}
$$

Submitting (12)-(16) into (11) yields that

$$
\begin{aligned}
& \dot{V}(t) \\
\leq & e^{\mathrm{T}}(t)\left(I_{N} \otimes L\right) e(t)+c e^{\mathrm{T}}(t)\left(G^{s} \otimes \Gamma\right) e(t)+\sum_{i=1}^{N}\left\|e_{i}\right\|_{2} \mu(t)+2 \sum_{i=1}^{N}\left\|e_{i}(t)\right\|_{2} h_{\max } \\
& +\sum_{i=1}^{N} e_{i}^{\mathrm{T}}(t)\left\{-d_{i} e_{i}(t)-\eta(t) \overline{\operatorname{sign}}\left(e_{i}(t)\right)-k \operatorname{sign}\left(e_{i}(t)\right)\left|e_{i}(t)\right|^{\beta}\right\} \\
= & W_{1}(t)+W_{2}(t)+W_{3}(t),
\end{aligned}
$$

where

$$
\begin{aligned}
& W_{1}(t)=e^{\mathrm{T}}(t)\left(I_{N} \otimes L\right) e(t)+c e^{\mathrm{T}}(t)\left(G^{s} \otimes \Gamma\right) e(t)-\sum_{i=1}^{N} e_{i}^{\mathrm{T}}(t) d_{i} e_{i}(t), \\
& W_{2}(t)=\sum_{i=1}^{N}\left\|e_{i}\right\|_{2} \mu(t)+\sum_{i=1}^{N}\left\|e_{i}(t)\right\|_{2}\left(2 h_{\max }\right)-\sum_{i=1}^{N} e_{i}^{\mathrm{T}}(t)\left(\eta(t) \overline{\operatorname{sign}}\left(e_{i}(t)\right)\right), \\
& W_{3}(t)=-\sum_{i=1}^{N} e_{i}^{\mathrm{T}}(t) k \operatorname{sign}\left(e_{i}(t)\right)\left|e_{i}(t)\right|^{\beta} .
\end{aligned}
$$

By virtue of Lemma 3.2 and (9), it is known that

$$
\begin{aligned}
W_{1}(t) & =e^{\mathrm{T}}(t)\left(I_{N} \otimes L\right) e(t)+c e^{\mathrm{T}}(t)\left(G^{s} \otimes \Gamma\right) e(t)-e^{\mathrm{T}}(t)\left(D \otimes I_{n}\right) e_{i}(t) \\
& =e^{\mathrm{T}}(t)\left(I_{N} \otimes L+c\left(G^{s} \otimes \Gamma\right)-D \otimes I_{n}\right) e(t) \\
& \leq 0
\end{aligned}
$$

Since $e_{i}^{\mathrm{T}}(t) \overline{\operatorname{sign}}\left(e_{i}(t)\right)=\left(e_{i 1}(t), e_{i 2}(t), \cdots, e_{i n}(t)\right)\left(\operatorname{sign}\left(e_{i 1}(t)\right), \operatorname{sign}\left(e_{i 2}(t)\right), \cdots, \operatorname{sign}\left(e_{i n}(t)\right)\right)^{\mathrm{T}}=$ $\sum_{j=1}^{n}\left|e_{i j}(t)\right|$ and $\left.\left\|e_{i}(t)\right\|_{2}-e_{i}^{\mathrm{T}}(t) \overline{\operatorname{sign}}\left(e_{i}(t)\right)\right)=\left(\sum_{j=1}^{n} e_{i j}^{2}(t)\right)^{\frac{1}{2}}-\sum_{j=1}^{n}\left|e_{i j}(t)\right| \leq 0$ according to Lemma 3.3, one can give

$$
W_{2}(t)=\sum_{i=1}^{N}\left(\mu(t)+2 h_{\max }\right)\left\|e_{i}(t)\right\|_{2}-\eta(t) \sum_{i=1}^{N} e_{i}^{\mathrm{T}}(t) \overline{\operatorname{sign}}\left(e_{i}(t)\right)
$$




$$
\begin{aligned}
& \leq\left(\mu(t)+2 h_{\max }\right) \sum_{i=1}^{N}\left\|e_{i}(t)\right\|_{2}-\eta(t) \sum_{i=1}^{N}\left\|e_{i}(t)\right\|_{2} \\
& =\left(\mu(t)+2 h_{\max }-\eta(t)\right) \sum_{i=1}^{N}\left\|e_{i}(t)\right\|_{2} \\
& \leq 0
\end{aligned}
$$

Considering that $\left|e_{i}(t)\right|=\left(\left|e_{i 1}(t)\right|,\left|e_{i 2}(t)\right|, \cdots,\left|e_{i n}(t)\right|\right)^{\mathrm{T}}$ and $\left|e_{i}(t)\right|^{\beta}=\left(\left|e_{i 1}(t)\right|^{\beta},\left|e_{i 2}(t)\right|^{\beta}, \cdots\right.$, $\left.\left|e_{i n}(t)\right|^{\beta}\right)^{\mathrm{T}}$, we have

$$
\begin{aligned}
W_{3}(t) & =-\sum_{i=1}^{N} e_{i}^{\mathrm{T}}(t) k \operatorname{sign}\left(e_{i}(t)\right)\left|e_{i}(t)\right|^{\beta} \\
& =-k \sum_{i=1}^{N}\left|e_{i}(t)\right|\left|e_{i}(t)\right|^{\beta} \\
& =-k \sum_{i=1}^{N} \sum_{j=1}^{n}\left|e_{i j}(t)\right|^{1+\beta}
\end{aligned}
$$

By Lemma 3.3, it can be obtained that

$$
\left(\sum_{i=1}^{N} \sum_{j=1}^{n}\left|e_{i j}(t)\right|^{1+\beta}\right)^{\frac{1}{1+\beta}} \geq\left(\sum_{i=1}^{N} \sum_{j=1}^{n}\left|e_{i j}(t)\right|^{2}\right)^{\frac{1}{2}},
$$

and

$$
\left(\sum_{i=1}^{N} \sum_{j=1}^{n}\left|e_{i j}(t)\right|^{1+\beta}\right) \geq\left(\sum_{i=1}^{N} \sum_{j=1}^{n}\left|e_{i j}(t)\right|^{2}\right)^{\frac{1+\beta}{2}}=\left(\sum_{i=1}^{N} e_{i}(t)^{\mathrm{T}} e_{i}(t)\right)^{\frac{1+\beta}{2}},
$$

which together with (20) implies that

$$
\begin{aligned}
W_{3}(t) & \leq-k\left(\sum_{i=1}^{N} e_{i}(t)^{\mathrm{T}} e_{i}(t)\right)^{\frac{1+\beta}{2}} \\
& =-k(2 V(t))^{\frac{1+\beta}{2}} \\
& =-2^{\frac{1+\beta}{2}} k V(t)^{\frac{1+\beta}{2}} .
\end{aligned}
$$

Submitting (18), (19) and (21) to (17), we can get

$$
\dot{V}(t) \leq W_{1}(t)+W_{2}(t)+W_{3}(t) \leq-2^{\frac{1+\beta}{2}} k V(t)^{\frac{1+\beta}{2}} .
$$

According to Lemma 3.1, $V(t)$ converges to zero in a finite time, and the finite time $t^{*}$ is given by

$$
t^{*}=\frac{V(0)^{1-0.5(1+\beta)}}{2^{\frac{1+\beta}{2}} k(1-0.5(1+\beta))}=\frac{V(0)^{\frac{1-\beta}{2}}}{\left.2^{\frac{\beta-1}{2}} k(1-\beta)\right)} .
$$

Hence, the error vector $e_{i}(t)$ converges to zero within $t^{*}$ for $i=1,2, \cdots, N$, and (1) under (6) is finite-timely synchronized in the finite time $t^{*}$. The proof is completed. 
Remark 3.5 Theorem 3.4 also provides a method how to select controllers for getting the finite-time synchronization of a linearly coupled heterogeneous complex network (1) when the Laplacian matrix $G$ is asymmetric and the nodes are nonidentical. The controller is divided into three parts: The first part $-\eta_{i} e_{i}(t)$ overcomes the linear condition of nonlinear function, the second part $-d(t) \overline{\operatorname{sign}}\left(e_{i}(t)\right)$ is used to offset the difference between state functions $f_{i}\left(t, x_{i}(t)\right)$ and the uncertain disturbance $h_{i}\left(t, x_{i}(t)\right)$, and the last one $-k \operatorname{sign}\left(e_{i}(t)\right)\left|e_{i}(t)\right|^{\beta}$ is used to make the network achieve the finite-time synchronization.

If the nodes are identical which indicates that $f_{1}, f_{2}, \cdots, f_{N}$ are equal to the same function denoted by $f$, then (1) becomes

$$
\dot{x}_{i}(t)=f\left(t, x_{i}\right)+h_{i}\left(t, x_{i}(t)\right)+c \sum_{j=1}^{N} G_{i j} \Gamma x_{j}(t), \quad i=1,2, \cdots, N .
$$

The drive dynamical node with $\left.x_{0}\right|_{t=0}=x_{0}(0)$ is given by

$$
\dot{x_{0}}(t)=f\left(t, x_{0}(t)\right)+h_{0}\left(t, x_{0}(t)\right)
$$

and it can be seen that the error dynamical system is

$$
\dot{e}_{i}(t)=F\left(t, e_{i}(t)\right)+H_{i}\left(t, e_{i}(t)+c \sum_{j=1}^{N} G_{i j} \Gamma e_{j}+u_{i}(t),\right.
$$

where $F_{i}(t)=f\left(t, x_{i}(t)\right)-f\left(t, x_{0}(t)\right)$ and $H_{i}\left(t, e_{i}(t)\right)=h_{i}\left(t, x_{i}(t)\right)-h_{0}\left(t, x_{0}(t)\right)$.

Let

$$
u_{i}(t)=-d_{i} e_{i}(t)-\eta(t) \overline{\operatorname{sign}}\left(e_{i}(t)\right)-k \operatorname{sign}\left(e_{i}(t)\right)\left|e_{i}(t)\right|^{\beta}, \quad i=1,2, \cdots, N,
$$

where $d_{i} \geq 0$ is constant to be determined for $i=1,2, \cdots, N ; k>0$ is a constant; $\overline{\operatorname{sign}}\left(e_{i}(t)\right)=$ $\left(\operatorname{sign}\left(e_{i 1}(t)\right), \operatorname{sign}\left(e_{i 2}(t)\right), \cdots, \operatorname{sign}\left(e_{i n}(t)\right)\right)^{\mathrm{T}},\left|e_{i}(t)\right|^{\beta}=\left(\left|e_{i 1}(t)\right|^{\beta},\left|e_{i 2}(t)\right|^{\beta}, \cdots,\left|e_{i n}(t)\right|^{\beta}\right)^{\mathrm{T}}, \operatorname{sign}$ $\left(e_{i}(t)\right)=\operatorname{diag}\left(\operatorname{sign}\left(e_{i 1}(t)\right), \operatorname{sign}\left(e_{i 2}(t)\right), \cdots, \operatorname{sign}\left(e_{i n}(t)\right)\right)$; the real number $\beta$ satisfies $0 \leq \beta<1$. Then, we can get a criterion on the finite-time synchronization of the complex network with identical nodes.

Corollary 3.6 Consider the complex network (22) under the set of controllers (24). If Assumption 2.1 and Assumption 2.3 hold, and

$$
\eta(t) \geq h_{\max }, \quad I_{N} \otimes L+c\left(G^{s} \otimes \Gamma\right)-D \otimes I_{n}<0
$$

with $D=\operatorname{diag}\left(d_{1}, d_{2}, \cdots, d_{N}\right)>0$, then (22) can be synchronized to the state of the node (23) in a finite time

$$
t^{*}=\frac{V(0)^{\frac{1-\beta}{2}}}{\left.2^{\frac{\beta-1}{2}} k(1-\beta)\right)} .
$$

Furthermore, if $h_{i}=0$ for $i=0,1, \cdots, N$ in $(22)$, that is, all nodes not only are identical but also have no uncertain disturbance, then (1) is reduced to

$$
\dot{x}_{i}(t)=f\left(t, x_{i}(t)\right)+c \sum_{j=1}^{N} G_{i j} \Gamma x_{j}(t),
$$


and the drive dynamical node of the complex network model (25) with $\left.x_{0}\right|_{t=0}=x_{0}(0)$ is

$$
\dot{x_{0}}(t)=f\left(t, x_{0}(t)\right) \text {. }
$$

In this case, the finite-time synchronization of (7) is changed to the problem of the complex network (25) with identical nodes. A criteria for the finite-time synchronization is given in the corollary below.

Corollary 3.7 Consider the complex network (25) under the controllers

$$
u_{i}(t)=-d_{i} e_{i}(t)-k \operatorname{sign}\left(e_{i}(t)\right)\left|e_{i}(t)\right|^{\beta}, \quad i=1,2, \cdots, N,
$$

where $k$ is a positive constant, $0 \leq \beta<1,\left|e_{i}(t)\right| \beta=\left(\left|e_{i 1}(t)\right|^{\beta},\left|e_{i 2}(t)\right|^{\beta}, \cdots,\left|e_{i n}(t)\right|^{\beta}\right)^{\mathrm{T}}$ and $\operatorname{sign}\left(e_{i}(t)\right)=\operatorname{diag}\left(\operatorname{sign}\left(e_{i 1}(t)\right), \operatorname{sign}\left(e_{i 2}(t)\right), \cdots, \operatorname{sign}\left(e_{i n}(t)\right)\right)$. If Assumption 2.1 holds and

$$
I_{N} \otimes L+c\left(G^{s} \otimes \Gamma\right)-D \otimes I_{n}<0
$$

with $D=\operatorname{diag}\left(d_{1}, d_{2}, \cdots, d_{N}\right)>0$, then (25) can be synchronized in a finite time

$$
t^{*}=\frac{V(0)^{\frac{1-\beta}{2}}}{\left.2^{\frac{\beta-1}{2}} k(1-\beta)\right)} .
$$

\section{Adaptive Finite-Time Synchronization}

This section discusses the adaptive finite-time synchronization of (1). We design an adaptive controllers as follows

$$
\begin{aligned}
& u_{i}(t)=-c d_{i}(t) \Gamma e_{i}(t)-\eta(t) \overline{\operatorname{sign}}\left(e_{i}(t)\right)-k \operatorname{sign}\left(e_{i}(t)\right)\left|e_{i}(t)\right|^{\beta}, \\
& \dot{d}_{i}(t)=q_{i} e_{i}^{\mathrm{T}}(t) \Gamma e_{i}(t)-k\left(\frac{c}{q_{i}}\right)^{\frac{\beta-1}{2}} \operatorname{sign}\left(d_{i}(t)-d\right)\left|d_{i}(t)-d\right|^{\beta},
\end{aligned}
$$

where $d$ is a constant to be determined; $k>0$ is a constant; $\left|e_{i}(t)\right|^{\beta}=\left(\left|e_{i 1}(t)\right|^{\beta},\left|e_{i 2}(t)\right|^{\beta}\right.$, $\left.\cdots,\left|e_{i n}(t)\right|^{\beta}\right)^{\mathrm{T}}, \overline{\operatorname{sign}}\left(e_{i}(t)\right)=\left(\operatorname{sign}\left(e_{i 1}(t)\right), \cdots, \operatorname{sign}\left(e_{i n}(t)\right)\right)^{\mathrm{T}}, \operatorname{sign}\left(e_{i}(t)\right)=\operatorname{diag}\left(\operatorname{sign}\left(e_{i 1}(t)\right)\right.$, $\left.\cdots, \operatorname{sign}\left(e_{i n}(t)\right)\right)$; the real number $\beta$ satisfies $0 \leq \beta<1$.

Theorem 4.1 Consider the complex network (1) under the set of adaptive controllers (26). If Assumption 2.1, Assumption 2.2 and Assumption 2.3 hold, and

$$
\eta(t) \geq 2 h_{\max }, \quad d \geq \frac{1}{c} \lambda_{\max }\left(I_{N} \otimes\left(L \Gamma^{-1}\right)+c G^{s} \otimes I_{n}\right),
$$

then (22) can be synchronized in a finite time

$$
t^{*}=\frac{V(0)^{\frac{1-\beta}{2}}}{\left.2^{\frac{\beta-1}{2}} k(1-\beta)\right)},
$$

where $\lambda_{\max }(\cdot)$ represents the largest eigenvalue of a matrix, $V(0)=\frac{1}{2} \sum_{i=1}^{N} e_{i}^{\mathrm{T}}(0) e_{i}(0)+$ $\sum_{i=1}^{N} \frac{c}{2 q_{i}}\left(d_{i}(0)-d\right)^{2}$, and $e_{i}(0)$ is the initial condition of $e_{i}(t)=x_{i}(t)-x_{0}(t)$ for $i=1,2, \cdots, N$. 
Proof For (1) under (26), a Lyapunov function is constructed as

$$
V(t)=\frac{1}{2} \sum_{i=1}^{N} e_{i}^{\mathrm{T}}(t) e_{i}(t)+\sum_{i=1}^{N} \frac{c}{2 q_{i}}\left(d_{i}(t)-d\right)^{2} .
$$

Along the trajectory of the error system (8), the derivative of $V(t)$ can be

$$
\begin{aligned}
\dot{V}(t)= & \sum_{i=1}^{N} e_{i}^{\mathrm{T}}(t) \dot{e}_{i}(t)+\sum_{i=1}^{N} \frac{c}{q_{i}}\left(d_{i}(t)-d\right) d_{i}(t) \\
= & \sum_{i=1}^{N} e_{i}^{\mathrm{T}}(t)\left\{F_{i}\left(t, e_{i}(t)\right)+H_{i}\left(t, e_{i}(t)\right)+c \sum_{j=1}^{N} G_{i j} \Gamma e_{j}(t)+u_{i}(t)\right\} \\
& +\sum_{i=1}^{N} \frac{c}{q_{i}}\left(d_{i}(t)-d\right)\left\{q_{i} e_{i}^{\mathrm{T}}(t) \Gamma e_{i}(t)-k\left(\frac{c}{q_{i}}\right)^{\frac{\beta-1}{2}} \operatorname{sign}\left(d_{i}(t)-d\right)\left|d_{i}(t)-d\right|^{\beta}\right\} \\
= & \sum_{i=1}^{N} e_{i}^{\mathrm{T}}(t) F_{i}\left(t, e_{i}(t)\right)+\sum_{i=1}^{N} e_{i}^{\mathrm{T}}(t) H_{i}\left(t, e_{i}(t)\right)+c \sum_{i=1}^{N} e_{i}^{\mathrm{T}}(t) \sum_{j=1}^{N} G_{i j} \Gamma e_{j}(t) \\
& +\sum_{i=1}^{N} e_{i}^{\mathrm{T}}(t)\left\{-c d_{i}(t) \Gamma e_{i}(t)-\eta(t) \overline{\operatorname{sign}}\left(e_{i}(t)\right)-k \operatorname{sign}\left(e_{i}(t)\right)\left|e_{i}(t)\right|^{\beta}\right\} \\
& +c \sum_{i=1}^{N}\left(d_{i}(t)-d\right) e_{i}^{\mathrm{T}}(t) \Gamma e_{i}(t) \\
& \left.-\sum_{i=1}^{N} k\left(\frac{c}{q_{i}}\right)^{\frac{1+\beta}{2}}\left(d_{i}(t)-d\right) \operatorname{sign}\left(d_{i}(t)-d\right)\left|d_{i}(t)-d\right|^{\beta}\right) \\
= & W_{1}(t)+W_{2}(t)+W_{3}(t),
\end{aligned}
$$

where

$$
\begin{aligned}
W_{1}(t)= & \sum_{i=1}^{N} e_{i}^{\mathrm{T}}(t) F_{i}\left(t, e_{i}(t)\right)+c \sum_{i=1}^{N} e_{i}^{\mathrm{T}}(t) \sum_{j=1}^{N} G_{i j} \Gamma e_{j}(t) \\
& -\sum_{i=1}^{N} e_{i}^{\mathrm{T}}(t) c d_{i}(t) \Gamma e_{i}(t)+c \sum_{i=1}^{N}\left(d_{i}(t)-d\right) e_{i}^{\mathrm{T}}(t) \Gamma e_{i}(t), \\
W_{2}(t)= & \sum_{i=1}^{N} e_{i}^{\mathrm{T}}(t) H_{i}\left(t, e_{i}(t)\right)-\sum_{i=1}^{N} e_{i}^{\mathrm{T}}(t)\left(\eta(t) \overline{\operatorname{sign}}\left(e_{i}(t)\right)\right), \\
W_{3}(t)= & -\sum_{i=1}^{N} e_{i}^{\mathrm{T}}(t) k \operatorname{sign}\left(e_{i}(t)\right)\left|e_{i}(t)\right|^{\beta} \\
& \left.-\sum_{i=1}^{N} k\left(\frac{c}{q_{i}}\right)^{\frac{1+\beta}{2}}\left(d_{i}(t)-d\right) \operatorname{sign}\left(d_{i}(t)-d\right)\left|d_{i}(t)-d\right|^{\beta}\right) .
\end{aligned}
$$


Letting $d \geq \frac{1}{c} \lambda_{\max }\left(I_{N} \otimes\left(L \Gamma^{-1}\right)+c G^{s} \otimes I_{n}\right)$, and similar to the proof of Theorem 3.4, we can obtain

$$
\begin{aligned}
W_{1}(t) & =e^{\mathrm{T}}(t)\left(I_{N} \otimes L\right) e(t)+c e^{\mathrm{T}}(t)\left(G^{s} \otimes \Gamma\right) e(t)-c \sum_{i=1}^{N} e_{i}^{\mathrm{T}}(t) d \Gamma e_{i}(t) \\
& =e^{\mathrm{T}}(t)\left(I_{N} \otimes L+c\left(G^{s}-d I_{N}\right) \otimes \Gamma\right) e(t) \leq 0 \\
W_{2}(t) & =\sum_{i=1}^{N}\left\|e_{i}(t)\right\|_{2}\left(2 h_{\max }\right)-\sum_{i=1}^{N} e_{i}^{\mathrm{T}}(t)\left(\eta(t) \overline{\operatorname{sign}}\left(e_{i}(t)\right)\right) \\
& \leq \eta(t)\left(\sum_{i=1}^{N}\left\|e_{i}(t)\right\|_{2}-\sum_{i=1}^{N}\left|e_{i}(t)\right|\right) \\
& \leq 0
\end{aligned}
$$

and

$$
\begin{aligned}
W_{3}(t) & =-k\left(\sum_{i=1}^{N}\left|e_{i}(t)\right|^{1+\beta}+\sum_{i=1}^{N}\left(\frac{c}{q_{i}}\right)^{\frac{1+\beta}{2}}\left|d_{i}(t)-d\right|^{1+\beta}\right) \\
& \leq-k\left(\sum_{i=1}^{N}\left\|e_{i}(t)\right\|_{2}^{2}+\sum_{i=1}^{N} \frac{c}{q_{i}}\left|d_{i}(t)-d\right|^{2}\right)^{\frac{1+\beta}{2}} \\
& =-2^{\frac{1+\beta}{2}} k V(t)^{\frac{1+\beta}{2}} .
\end{aligned}
$$

Submitting (29)-(31) into (28), we can get

$$
\dot{V}(t) \leq W_{1}(t)+W_{2}(t)+W_{3}(t) \leq-2^{\frac{1+\beta}{2}} k V(t)^{\frac{1+\beta}{2}} .
$$

By Lemma 3.1, $V(t)$ converges to zero in a finite time, and the finite time $t^{*}$ is given by

$$
t^{*}=\frac{V(0)^{1-0.5(1+\beta)}}{2^{\frac{1+\beta}{2}} k(1-0.5(1+\beta))}=\frac{V(0)^{\frac{1-\beta}{2}}}{\left.2^{\frac{\beta-1}{2}} k(1-\beta)\right)} .
$$

Hence, the error vector $e_{i}(t), i=1,2, \cdots, N$, can converge to 0 within $t^{*}$. Consequently, under the controllers (26), the complex network (1) is synchronized in the finite time $t^{*}$. The proof is completed.

\section{Numerical Example}

Consider a five-pendulum coupled nonlinear system ${ }^{[37]}$ with linearly and diffusively coupling in which the dynamics of the $i$ th node is described by

$$
\dot{x}_{i}(t)=f_{i}\left(t, x_{i}\right)+h_{i}\left(t, x_{i}\right)+c \sum_{j=1}^{5} G_{i j} \Gamma x_{j}(t), \quad i=1,2, \cdots, 5
$$

with initial values $X(0)=\left(x_{1}^{\mathrm{T}}(0), x_{2}^{\mathrm{T}}(0), \cdots, x_{5}^{\mathrm{T}}(0)\right)^{\mathrm{T}}=(0.60 .91 .2-0.3-0.90-1.21 .51 .05-$ $1.2)^{\mathrm{T}}$. The inner-coupling matrix $\Gamma$ and the Laplacian matrix $G$ are 


$$
\Gamma=\left[\begin{array}{ll}
1 & 0 \\
0 & 0
\end{array}\right], \quad G=\left[\begin{array}{ccccc}
-1 & 1 & 0 & 0 & 0 \\
0 & -2 & 2 & 0 & 0 \\
0 & 0 & -2 & 2 & 0 \\
0 & 0 & 0 & -1 & 1 \\
0 & 0 & 0 & 0 & 0
\end{array}\right] .
$$

Its activation function $f_{i}\left(t, x_{i}(t)\right)=\left(x_{i 2},-q x_{i 2}-r_{i} \sin \left(x_{i 1}\right)\right)^{\mathrm{T}}$ and its uncertain disturbance $h_{i}\left(t, x_{i}\right)=\left(0, \phi_{i}(t)\right)^{\mathrm{T}}$, where $q=3.15, r_{i}=0.02$ for $i=1,2, \cdots, 5$, and $\left(\phi_{1}, \phi_{2}, \cdots, \phi_{5}\right)^{\mathrm{T}}=$ $(0.1 \sin (t),-0.15 \sin (t),-0.2 \sin (t), 0.15 \sin (t), 0.2 \sin (t))^{\mathrm{T}}$. The drive dynamical node is

$$
\dot{x}_{0}(t)=f_{0}\left(t, x_{i}\right)+h_{0}\left(t, x_{i}\right)
$$

with initial values $x_{0}(0)=(1,1)^{\mathrm{T}}$, where $f_{0}\left(t, x_{0}\right)=\left(x_{02},-q x_{02}\right)^{\mathrm{T}}, h_{0}\left(t, x_{0}\right)=(0,0)^{\mathrm{T}}$, and the coupling strength $c=2.0$.

As a comparison, the state response trajectory and the state error response track of the heterogeneous complex dynamic network (32) without control are given. It can be seen from Figure 1 that before the application of the control the synchronization errors of each node do not tend to 0 , and then the states of nodes have not been synchronized.

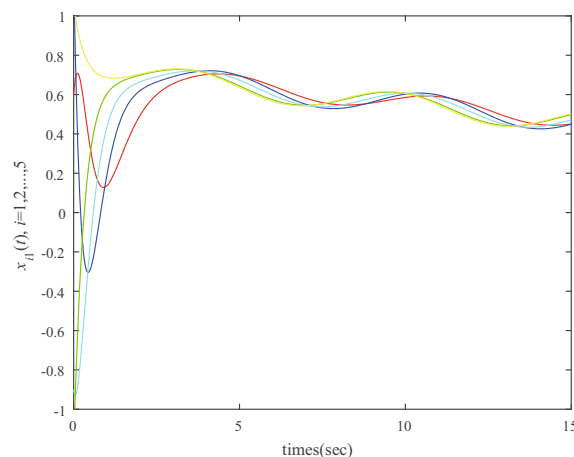

(a) State response $x_{i 1}$

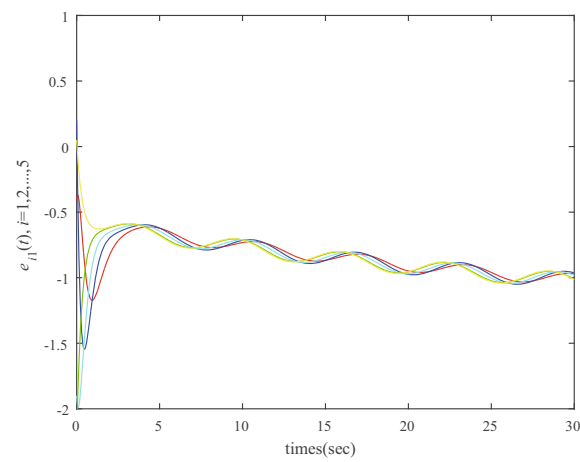

(c) Synchronization error $e_{i 1}$

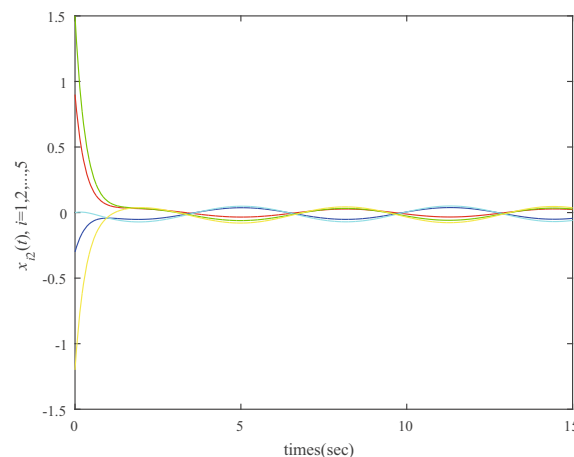

(b) State response $x_{i 2}$

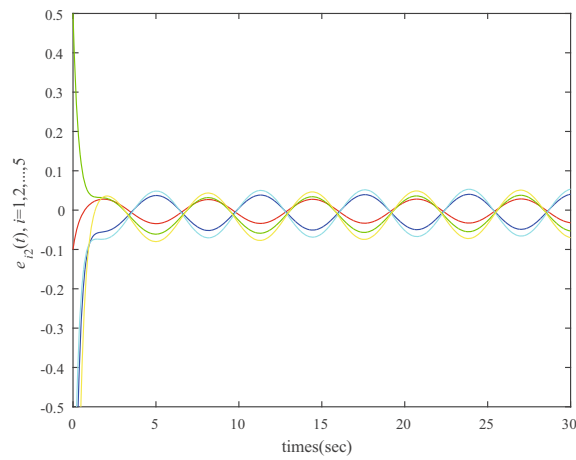

(d) Synchronization error $e_{i 2}$

Figure 1 State responses and synchronization error without control 
Let $L=I_{2}, \mu(t)=0.1$ and $h_{\max }=0.5$, which implies that Assumption 2.1, Assumption 2.2 and Assumption 2.3 hold since $\left\|f_{i}(t, x)-f_{0}(t, x)\right\|=\|\left(0,-r_{i} \sin \left(x_{1}\right) \|=r_{i} \leq 0.1\right.$. With $D=\operatorname{diag}(2,2,2,2,2)$, it is known that $\lambda_{\max }\left(I_{N} \otimes L+c\left(G^{s} \otimes \Gamma\right)-D \otimes I_{n}\right)=-0.3592$, which indicates that (9) can be satisfied. According to Theorem 3.4 with $\eta(t)=\mu(t)+h_{\max }=0.6, k=$ $1, \beta=0.6$, by use of (6), (32) can be synchronized to the drive node (33). From Figure 2 , it can be seen that the synchronization error of each node is reduced to 0 within $t^{*}=4.3766$, and the node state is synchronized within $t^{*}=4.3766$. In fact, the time of the numerical simulation actually synchronizes is 0.6090 seconds.

Furthermore, we use (26) with $d=5$ and the initial values $X(0)=\left[x_{1}^{\mathrm{T}}(0), X_{2}^{\mathrm{T}}(0), x_{3}^{\mathrm{T}}(0)\right.$,

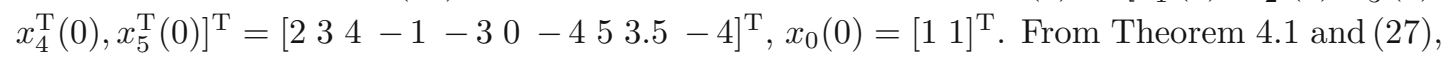
it can be seen that $(32)$ can be synchronized within the finite time $t^{*}=10.4051$ and the actual synchronization time is 0.1074 seconds, which is illustrated by Figure 3 .

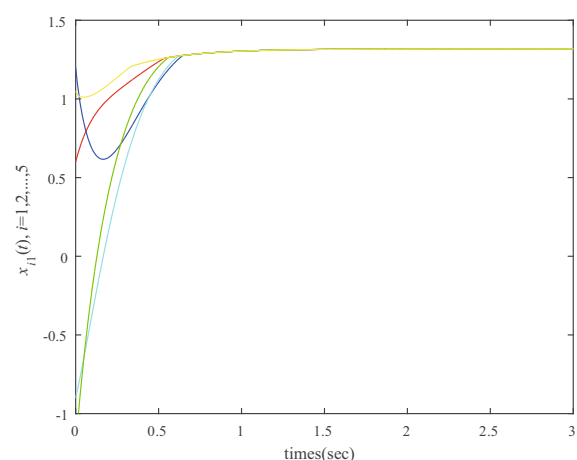

(a) State response $x_{i 1}$

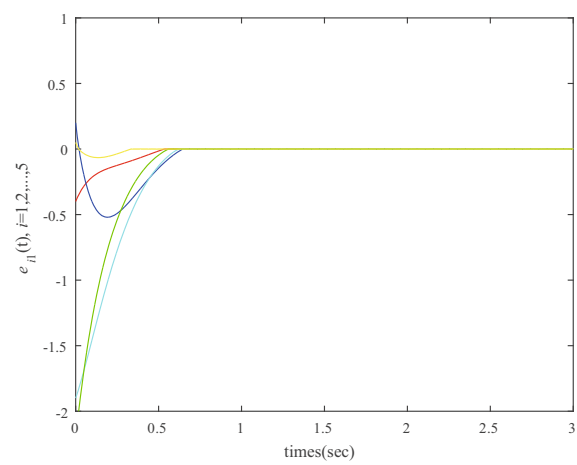

(c) Synchronization error $e_{i 1}$

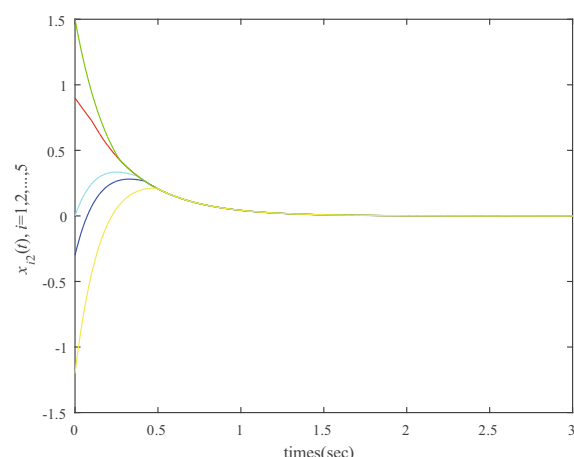

(b) State response $x_{i 2}$

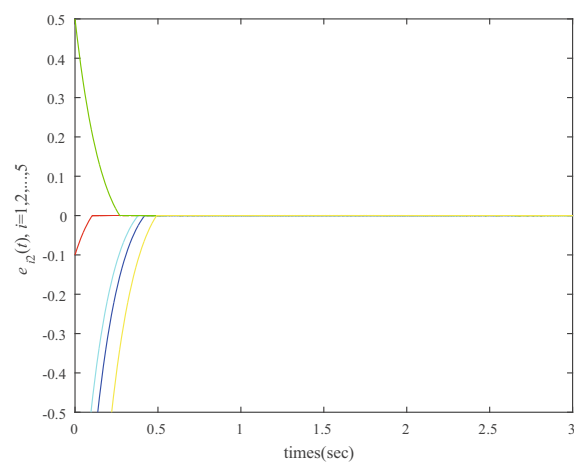

(d) Synchronization error $e_{i 2}$

Figure 2 Finite-time synchronization 


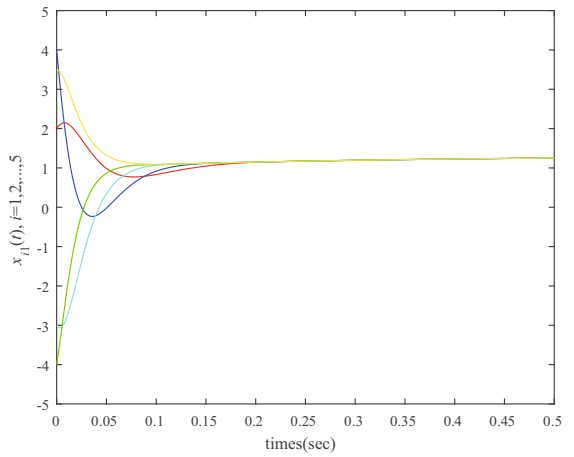

(a) State response $x_{i 1}$

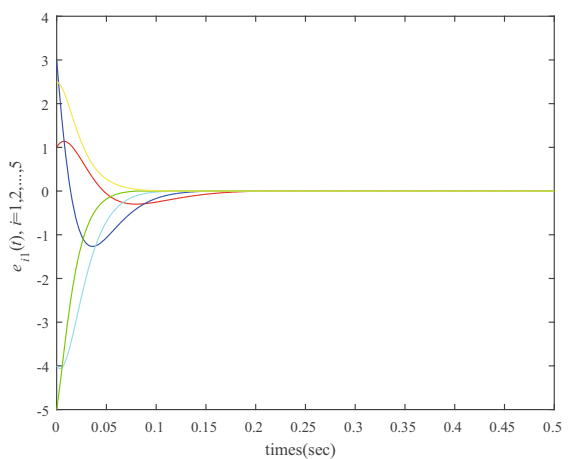

(c) Synchronization error $e_{i 1}$

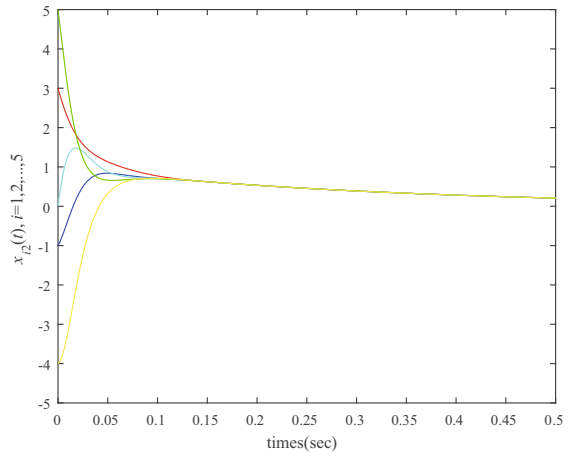

(b) State response $x_{i 2}$

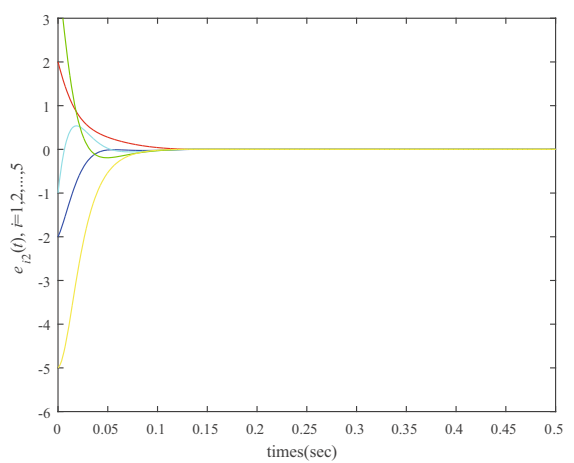

(d) Synchronization error $e_{i 2}$

Figure 3 Adaptive finite-time synchronization

\section{Conclusions}

This paper has discussed the finite-time synchronization for a class of dynamical complex network with nonidentical nodes and uncertain disturbance, some sufficient conditions have been proposed by using the Lyapunov function method and the linear matrix inequality technique, and they have been applied to the case of identical nodes and the one without uncertain disturbance. Also the adaptive finite-time synchronization has been studied. Future work can focus on the fixed-time synchronization for complex networks with nonidentical nodes and uncertain disturbance.

\section{References}

[1] Yu W, Chen G, and Lü J, On pinning synchronization of complex dynamical networks, Automatica, 2009, 45(2): 429-435. 
[2] Wu C W, Synchronization in Complex Networks of Nonlinear Dynamical Systems, World Scientific, Singapore, 2007.

[3] Osipov G, Kurths J, and Zhou C, Synchronization in Oscillatory Networks, Springer, Berlin, 2007.

[4] Arenas A, Daz-Guilera A, Kurths J, et al., Synchronization in complex networks, Physics Reports, 2008, 469: 93-153.

[5] Song Q and Cao J D, On pinning synchronization of directed and undirected complex dynamical networks, IEEE Trans. Circuits Syst. I., 2010, 57: 672-680.

[6] Wu Y Y, Cao J D, Alofic A, et al., Finite-time boundedness and stabilization of uncertain switched neural networks with time-varying delay, Neural Networks, 2015, 69: 135-143.

[7] Ma C and Zhang J, On formability of linear continuous multi-agent systems, Journal of Systems Science and Complexity, 2012, 25(1): 13-29.

[8] Zhou J, Lu J, and Lü J, Pinning adaptive synchronization of a general complex dynamical network, Automatica, 2008, 44(4): 996-1003.

[9] Ding Z and Li Z, Distributed adaptive consensus control of nonlinear output-feedback systems on directed graphs, Automatica, 2016, 72: 46-52.

[10] Xia W and Cao J, Pinning synchronization of delayed dynamical networks via periodically intermittent control, Chaos, 2009, 19: 113-120.

[11] Cai S M, Liu Z R, Xu F D, et al., Periodically intermittent controlling complex dynamcial networks with time-varying delays to a desired orbit, Phys. Lett. A, 2009, 373: 3846-3854.

[12] Liu X and Chen T, Cluster synchronization in directed networks via intermittent pinning control, IEEE Transactions on Neural Networks, 2011, 22(7): 1009-1020.

[13] Li H, Hu C, Jiang H, et al., Synchronization of fractional-order complex dynamical networks via periodically intermittent pinning control, Chaos Solitons and Fractals, 2017, 103: 357-363.

[14] Zhang Q, Lu J, and Zhao J, Impulsive synchronization of general continuous and discrete-time complex dynamical networks, Commun. Nonlinear Sci. Numer. Simul., 2010, 15: 1063-1070.

[15] Yang X, Cao J D, and Lu J Q, Synchronization of delayed complex dynamical networks with impulsive and stochastic effects, Nonlinear Anal. RWA, 2011, 12: 2252-2266.

[16] Gong X, Gan L, and Wu Z, Adaptive impulsive cluster synchronization in community network with nonidentical nodes, International Journal of Modern Physics C, 2016, 27(1): 1650010.

[17] He W L, Qian F, Lam J, et al., Quasi-synchronization of heterogeneous dynamic networks via distributed impulsive control: Error estimation, optimization and design, Automatica, 2015, 62: 249-262.

[18] Chen X Y, Park Ju H, Cao J D, et al., Sliding mode synchronization of multiple chaotic systems with uncertainties and disturbances, Applied Mathematics and Computation, 2017, 308: 161-173.

[19] Lü L, Yu M, Li C, et al., Projective synchronization of a class of complex network based on high-order sliding mode control, Nonlinear Dynamics, 2013, 73(1-2): 411-416.

[20] Hou H, Zhang Q, and Zheng M, Cluster synchronization in nonlinear complex networks under sliding mode control, Nonlinear Dynamics, 2016, 83(12): 739-749.

[21] Wang Y W, Xiao J W, Wen C, et al., Synchronization of continuous dynamical networks with discrete-time communications, IEEE Trans. Neural Netw., 2011, 22(120): 1979-1986.

[22] Wu Z, Park J, Su H, et al., Exponential synchronization for complex dynamical networks with sampled-data, Journal Franklin I, 2012, 349(9): 2735-2749.

[23] Zhao J, Hill D, Liu T, et al., Stability of dynamical networks with nonidentical nodes: A multiple 
V-Lyapunov function method, Automatica, 2011, 47: 2615-2625.

[24] Song Q, Cao J D, and Liu F, Synchronization of complex dynamical networks with nonidentical nodes, Physics Letters A, 2010, 374(4): 544-551.

[25] Wang S, Yao H, Zheng S, et al., A novel criterion for cluster synchronization of complex dynamical networks with coupling time-varying delays, Communications in Nonlinear Science and Numerical Simulation, 2012, 17(7): 2997-3004.

[26] Yang X, Wu Z, and Cao J, Finite-time synchronization of complex networks with nonidentical discontinuous nodes, Nonlinear Dynamics, 2013, 73(4): 2313-2327.

[27] Lu J Q, Ho D W, Cao J D, et al., Exponential synchronization of linearly coupled neural networks with impulsive disturbances, IEEE Trans Neural Netw., 2011, 22(2): 329-336.

[28] Cai G, Jiang S, Cai S, et al., Cluster synchronization of overlapping uncertain complex networks with time-varying impulse disturbances, Nonlinear Dynamics, 2015, 80(1-2): 503-513.

[29] Jiang S, Lu X, Cai G, et al., Adaptive fixed-time control for cluster synchronisation of coupled complex networks with uncertain disturbances, International Journal of Systems Science, 2017, 48(16): 1-9.

[30] Ma C, Li T, and Zhang J, Consensus control for leader-following multi-agent systems with measurement noises, Journal of Systems Science and Complexity, 2010, 23(1): 35-49.

[31] Yang X and Cao J, Finite-time stochastic synchronization of complex networks, Appl. Math. Model, 2010, 34(11): 3631-3641.

[32] Chen Y G, Fei S M, and Li Y M, Robust stabilization for uncertain saturated time-delay systems: A distributed-delay-dependent polytopic approach, IEEE Transactions on Automatic Control, 2017, 62(7): 3455-3460.

[33] Zong G D, Ren H L, and Hou L L, Finite-time stability of interconnected impulsive switched systems, IET Control Theory and Applications, 2016, 10(6): 648-654.

[34] Zong G D, Wang R H, Zheng W X, et al., Finite-time $H_{\infty}$ control for discrete-time switched nonlinear systems with time delay, International Journal of Robust and Nonlinear Control, 2015, 25(6): 914-936.

[35] Langville A N and Stewart W J, The Kronecker product and stochastic automata networks, J. Comput. Appl. Math., 2004, 16(7): 429-447.

[36] Wan Y, Cao J D, Wen G H, et al., Robust fixed-time synchronization of delayed Cohen-Grossberg neural networks, Neural Networks, 2016, 73: 86-94.

[37] Zhao J, Hill D J, and Liu T, Global bounded synchronization of general dynamical networks with nonidentical nodes, IEEE Transactions on Automatic Control, 2012, 57(10): 2656-2662. 